\title{
Oscillatory Dynamics in Bacterial Reaction Centres Studied by Electronic 2D Spectroscopy
}

\author{
D. Paleček ${ }^{1}$, S. Westenhoff ${ }^{2}$, P. Edlund ${ }^{2}$, P. Smith ${ }^{2}$ and D. Zigmantas ${ }^{2}$ \\ ${ }^{1}$ Department of Chemical Physics, Lund University, Getingevägen 60, 22100 Lund, Sweden \\ ${ }^{2}$ Department of Chemistry, University of Gothenburg, Medicinaregatan 9C, 40530 Gothenburg, \\ Sweden
}

\begin{abstract}
Coherent dynamics in bacterial reaction centres were studied by electronic $2 \mathrm{D}$ spectroscopy at $80 \mathrm{~K}$ temperature. Polarization measurements together with Fourier analysis allowed assignment of observed population time beatings at different frequencies to the coherences of electronic and vibrational character.
\end{abstract}

\section{Introduction}

Reaction centre (RC) is at the core of photosynthetic apparatus, where collected sunlight energy is converted into the electrochemical energy, which runs all the biological functions in photosynthetic organisms. Primary energy transfer pathways and rates in photosynthesis have been studied for many years within the framework of probabilistic population transfer. Only recently it has been reported that the energy could be transferred in the wavelike fashion [1,2]. This implies that quantum coherence could play a role in making initial photosynthetic function more robust and efficient.

In this work we investigated coherent dynamics in the RC from photosynthetic purple bacterium Rhodobacter $(\mathrm{Rb}$.) sphaeroides. The RC consists of bacteriochlorophyll dimer $(\mathrm{P})$ in the centre and two branches, each including one accessory bacteriochlorophyll (B) and one bacteriopheophytin $(\mathrm{H})$ molecule. In the experiments reported here, the primary electron donor $(\mathrm{P})$ is oxidized by the chemical oxidant $\mathrm{K} 3 \mathrm{Fe}(\mathrm{CN}) 6$. This prevents the electron transfer reactions and allows us to focus on the excitation energy transfer and coherence dynamics. Fleming and co-workers have reported a twocolour photon echo study on the oxidized RC [3]. In the experiment exploring the correlation between $\mathrm{H}$ and $\mathrm{B}$ absorption bands the authors observed the $250 \mathrm{~cm}^{-1}$ oscillation that persisted on the time scale of $\sim 400 \mathrm{fs}$. Using theoretical analysis oscillations were interpreted as originating from the cross-correlation between nuclear modes modulating B and $\mathrm{H}$ energy levels.

Here we present the polarization 2D spectroscopy study of the $\mathrm{RC}$ revealing the full picture of multiple frequency oscillations on the energy landscape covering $\mathrm{H}$ and $\mathrm{B}$ absorption bands.

\section{Results and Discussion}

$\mathrm{RCs}$ extracted from $\mathrm{Rb}$. sphaeroides were incubated with the chemical oxidant and then mixed with glycerol in the $40 \% / 60 \%$ proportion. All experiments were performed on cooled to $80 \mathrm{~K}$ sample in the liquid nitrogen flow cryostat. Broadband 15 fs laser pulses were used in the double modulation lock-in 2D spectroscopy setup described in [4]. The laser spectrum completely covered the absorption bands of $\mathrm{H}$ and B. Population times with 10 or $20 \mathrm{fs}$ steps were typically scanned for $1 \mathrm{ps.}$ 
To elucidate the origin of different coherent beatings, two polarization schemes were employed. In one of them polarizers in three excitation beams and detection polarizer were all set to parallel (0000 scheme) and in the other scheme polarizers were arranged at $\pi / 4,-\pi / 4, \pi / 2$ angles for the first, second and third pulses respectively. Whereas the polarizer in the detection channel was set to $0(\pi / 4$ $-\pi / 4 \pi / 20$ scheme). The latter scheme was proposed for suppressing the diagonal peaks and for exposing the cross-peaks in 2D IR spectroscopy [5]. Closer analysis of the $(\pi / 4-\pi / 4 \pi / 20)$ configuration reveals that the only signals, which do not vanish following orientational averaging are those that originate from the sequence of laser field interactions with transition dipoles, which have orientations as follows. The first and the second transition dipole moments have to be of different orientation, as well as the third and the fourth transitions. For electronic 2D spectroscopy it implies that only coherences with electronic character should be observed in this polarization scheme. In the remaining we will refer to these coherences as "excitonic". Very recently this polarization scheme was used to study electronic coherences in the light-harvesting II complexes from plants [6]. The ability to set the polarizers with high precision in our experiments is limited and the "leakage" from different polarization configuration is observed. We estimated that the suppression of the signals coming from other polarization orientations is $\sim 85$. Because electronic coherence signals are rather small, great care has to be taken to distinguish them from the "leaking" signals.
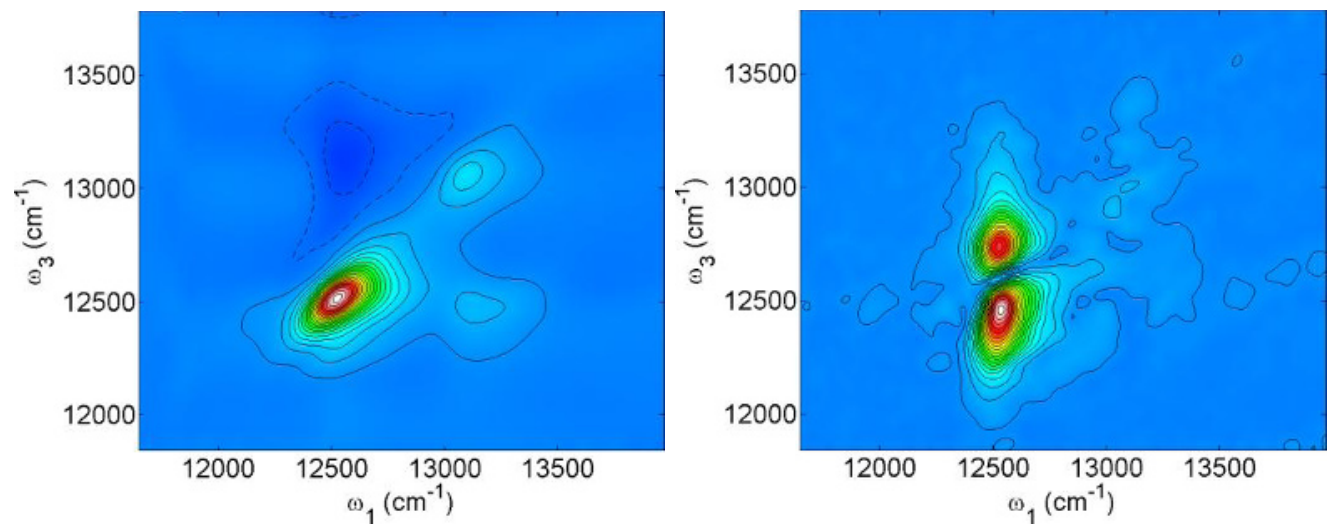

Fig. 1. Left the (0000) electronic $2 \mathrm{D}$ spectrum of the $\mathrm{RC}$ at $40 \mathrm{fs}$ population time. In the right panel the $2 \mathrm{D}$ Fourier amplitude of the $235 \mathrm{~cm}^{-1}$ component is plotted.

The real part of the (0000) 2D spectrum measured at 40 fs population time is show in Fig. 1. In the evolution of the $2 \mathrm{D}$ spectrum multiple beatings were observed on the time scale of a picosecond. To facilitate the analysis of contributions oscillating with different frequencies we fitted kinetics for each $\left(\omega_{1}, \omega_{3}\right)$ point, subtracted multi-exponential decays and performed Fourier transform along the population time in each point. In the right panel of the Fig. 1 Fourier amplitude of the most prominent low frequency component $\left(235 \mathrm{~cm}^{-1}\right)$ is shown. From the location of the Fourier amplitude in the 2D spectrum it is clear that these coherences originate from the vibrational wavepacket propagating in the electronic state of B. Furthermore by considering rephrasing and nonrephasing parts of the $2 \mathrm{D}$ spectra we were able to show that the observed wavepacket is located on the ground state.

In Fig. 2 the real part of the $(\pi / 4-\pi / 4 \pi / 20)$ 2D spectrum is shown (left) together with the Fourier amplitude of the $645 \mathrm{~cm}^{-1}$ frequency component at each $\left(\omega_{1}, \omega_{3}\right)$ point (right). This frequency corresponds exactly to the energy difference between the $\mathrm{H}$ and $\mathrm{B}$ absorption bands. Position and amplitude of the peaks match the ones expected from the exciton coherence signals. This together with the fact that the $(\pi / 4-\pi / 4 \pi / 20)$ polarization combination exposes electronic coherence signals implies that observed beatings originate from the electronic HB coherences. The other prominent oscillation frequency that exhibits features attributable to the coherences with excitonic character is $575 \mathrm{~cm}^{-1}$. This indicates that electronic and vibrational degrees of freedom are mixed and we observe beatings with mixed character. 

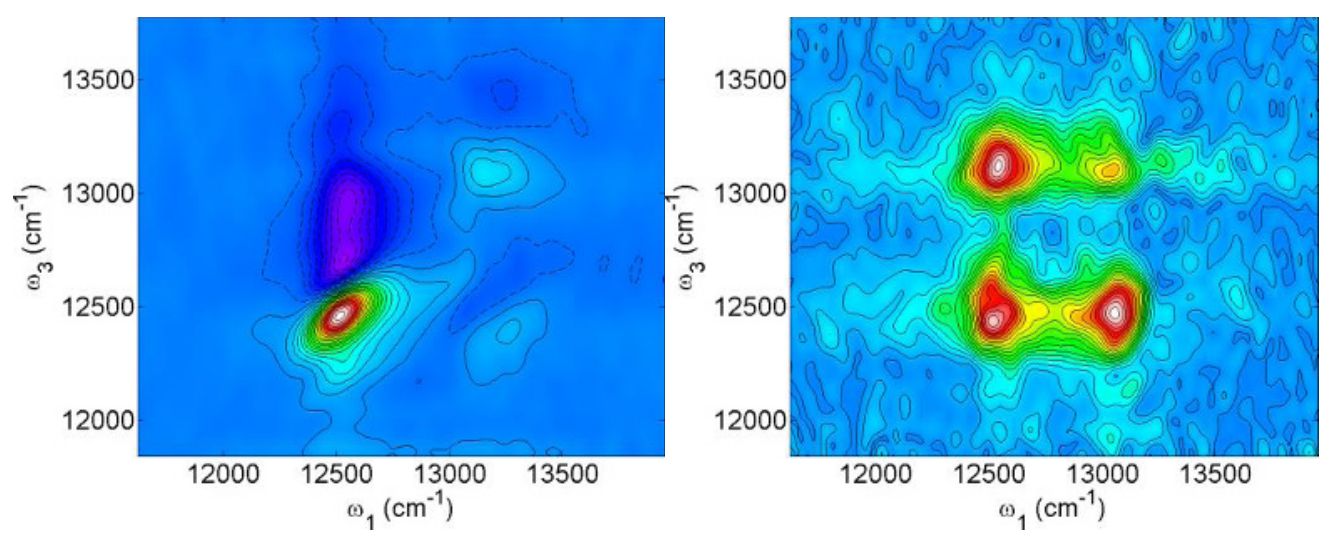

Fig. 2. Left the $(\pi / 4-\pi / 4 \pi / 20)$ electronic $2 \mathrm{D}$ spectrum of the $\mathrm{RC}$ at $40 \mathrm{fs}$ population time. In the right panel 2D Fourier amplitude of the $645 \mathrm{~cm}^{-1}$ component is plotted.

\section{Conclusions}

Combination of polarization 2D electronic spectroscopy experiments allowed distinction of coherences with excitonic and vibrational character in the oxidized bacterial RC. All the oscillating coherences observed at $80 \mathrm{~K}$ last on a picosecond time scale. Most oscillations originate from ground state vibrational wavepacket dynamics. In addition exciton coherences at the frequency matching exactly the energy gap between $\mathrm{H}$ and $\mathrm{B}$ absorption bands was also unravelled. Data suggest that excited state coherence dynamics involve mixing of electronic and nuclear degrees of freedom.

\section{References}

1. G. S. Engel, T. R. Calhoun, E. L. Read, T. K. Ahn, T. Mancal, Y. C. Cheng, G. R. Fleming, Nature 446, 782 (2007).

2. E. Collini, C. Y. Wong, K. E. Wilk, P. M. G. Curmi, P. Brumer, G. D. Scholes, Nature 463, 644 (2010).

3. H. Lee, Y. C. Cheng, G. R. Fleming, Science 316, 1462 (2007).

4. R. Augulis, D. Zigmantas, Opt Express 19, 13126 (2011).

5. M. T. Zanni, N. H. Ge, Y. S. Kim, R. M. Hochstrasser, Proc. Natl. Acad. Sci. USA 98, 11265 (2001).

6. G. S. Schlau-Cohen, A. Ishizaki, T. R. Calhoun, N. S. Ginsberg, M. Ballottari, R. Bassi, G. R. Fleming, Nature Chemistry 4, 389 (2012). 